\title{
Benefit from, and acclimatization to, frequency compression hearing aids in experienced adult hearing-aid users
}

Rachel Ellis and Kevin J. Munro

\section{Linköping University Post Print}

\section{Tweet}

N.B.: When citing this work, cite the original article.

Original Publication:

Rachel Ellis and Kevin J. Munro, Benefit from, and acclimatization to, frequency compression hearing aids in experienced adult hearing-aid users, 2015, International Journal of Audiology, (54), 1, 37-47.

http://dx.doi.org/10.3109/14992027.2014.948217

Copyright: Informa Healthcare http://informahealthcare.com/

Postprint available at: Linköping University Electronic Press

http://urn.kb.se/resolve?urn=urn:nbn:se:liu:diva-113490 
Ellis \& Munro, Frequency compression hearing aids

Benefit from, and acclimatisation to, frequency compression hearing aids in experienced adult hearing aid users

\author{
Rachel J. Ellis ${ }^{1,2}$ and Kevin J. Munro ${ }^{1,3}$ \\ ${ }^{1}$ School of Psychological Sciences, University of Manchester, UK \\ ${ }^{2}$ Linnaeus Centre HEAD, Swedish Institute for Disability Research, Department of Behavioural Sciences \\ and Learning, Linköping University, Sweden \\ ${ }^{3}$ Central Manchester University Hospitals NHS Foundation Trust, Manchester Academic Health Science \\ Centre, Manchester, U.K.
}

Key words: Frequency Compression, Auditory Acclimatisation, Speech Perception, Cognition, Sensorineural Hearing Loss.

Abbreviations
FC = Frequency Compression

Corresponding author: Rachel J. Ellis, Linnaeus Centre HEAD,

Swedish Institute for Disability Research

Department of Behavioural Sciences and Learning

Linköping University 58183

Sweden

Email: Rachel.Ellis@liu.se 
Ellis \& Munro, Frequency compression hearing aids

\section{ABSTRACT}

Objective: The aim was to investigate whether frequency compression (FC) hearing aids provide additional benefit to that conferred by conventional amplification.

Design: Participants wore the same hearing aid with FC enabled and disabled for six weeks (ABA design) in each condition. Speech recognition tests (in both quiet and in noise) were administered alongside two questionnaires. Performance was compared across the two signal processing conditions and at different time points.

Study sample: Twelve experienced hearing aid users (aged 65-84 years old) with moderate-to-severe high-frequency hearing loss participated in the study

Results: FC resulted in statistically significantly higher mean scores in all of the administered speech tests. No participant was significantly disadvantaged from the use of FC. Improvements over time were limited to high frequency phoneme perception. No effect of FC on self-report outcomes was observed.

Conclusions: FC may lead to significant improvements in speech perception outcomes in both quiet and noise for many individuals. No participant was significantly disadvantaged by the use of FC. 
Ellis \& Munro, Frequency compression hearing aids

Conventional hearing aids are often unable (due to bandwidth limitations or acoustic feedback) to amplify

high frequency sounds sufficiently to restore audibility. Alternative signal processing strategies, such as frequency lowering, have been developed to overcome this limitation. In hearing aids, frequency lowering is commonly accomplished using either frequency transposition (available as 'audibility extender' in some devices manufactured by Widex A/S, Denmark) or non-linear frequency compression (FC, available as 'sound recover' in some devices manufactured by Phonak AG, Switzerland). It is the latter method that was the focus of this study (see McDermott, 2011, for a detailed comparison of the two methods). FC is a method of altering the input signal such that frequencies above a given cut-off frequency are compressed into a reduced bandwidth (and amplified), resulting in the information being presented at a lower frequency. As the processing is non-linear, more compression is provided at the highest frequencies. Information below the cut-off frequency is amplified as normal and the compressed and non-compressed portions of the signal do not overlap.

Previous clinical studies of FC investigating benefit have produced mixed results with some listeners obtaining significant benefit and others showing no additional benefit (see for example, Simpson et al, 2005, 2006; Glista et al, 2009). Therefore, the primary aim of the present study was to investigate the degree, and time course, of additional benefit provided by FC compared to that provided by conventional amplification in experienced adult hearing aid users. The study also aimed to investigate perceptual changes over time resulting from the use of FC.

\section{Studies investigating frequency compression technology}

The first clinical study to investigate FC hearing aids was conducted by Simpson et al (2005). In this study, 17 adults with moderate-to-severe sloping hearing losses were fitted with prototype FC devices for four to six weeks. After this time, monosyllabic word recognition in quiet was assessed with both the FC enabled and disabled. The results showed that eight participants performed significantly better with FC, 
Ellis \& Munro, Frequency compression hearing aids

eight showed no difference and one participant performed significantly worse with FC. An analysis at the group level showed that frequency compression significantly improved detection of fricative and affricate consonants (with a mean improvement of approximately 11\%) without impairing the perception of vowels and mid frequency consonants. A second study by Simpson et al (2006) found no evidence of improvements in speech perception resulting from the use of FC in a sample of seven listeners with steeply sloping high frequency losses. On the surface, these results appear to suggest that listeners with a severely sloping loss are less likely to obtain benefit from FC than those with more moderately sloping losses. However, it is possible that the results were influenced by the fact that only four of the seven participants in this study were experienced hearing aid users compared to all of the listeners in the earlier (Simpson et al, 2005) study.

Glista et al (2009) also investigated benefit from FC using a sample of 24 participants with moderate-tosevere hearing loss, of which 13 were adults (aged 50-81 years old) and 11 were children (aged 6-17 years old). The participants were asked to wear the hearing aids as often as possible in their everyday lives (with and without FC being enabled). Performance with FC was measured on a number of speech tests and then compared to performance using the same hearing aids but with FC disabled. Speech tests included a series of phoneme recognition tests and a plural detection task. At an individual level, five adults and seven children showed a statistically significant improvement with FC on at least one of the outcome measures assessed. Self-reported preferences were also obtained, revealing that two of the adult participants favored the frequency compression setting along with seven of the child participants.

There are, however, a number of methodological issues which may influence the interpretation of these results. Much of the benefit reported is limited to improvement in speech sound detection and the plural recognition tasks. The improvement in detection thresholds for high frequency phonemes is unsurprising due to the signal processing strategy used in FC. Regarding improvements seen in the plural recognition 
Ellis \& Munro, Frequency compression hearing aids

task, it should be noted that the ability to determine whether a word is plural or not (as per the task used in this study) is simply indicative of the listeners ability to detect an additional phoneme. This does not necessarily mean that the listener was able to correctly recognize the phoneme or that they would be able to successfully discriminate between different high frequency sounds.

Another issue of interest is the discrepancy between the results of Simpson et al (2006) and Glista et al (2009). Simpson et al (2006) found no benefit of frequency compression to participants with a steeply sloping hearing loss. However, Glista et al (2009) found that these participants were the most likely to receive benefit from a frequency compression hearing aid. There are a number of possible reasons for this difference in findings. One explanation may relate to differences in the severity of high frequency hearing loss, participants in the Simpson et al (2006) study having poorer high frequency thresholds than those in the Glista et al (2009) study. The difference may also have been related to the outcome measures used or to the duration of time that the frequency compression aid was used, which was much longer in the later study. The studies also differed in how the frequency compression settings were determined.

Specifically, when fitting the hearing aid, Glista et al (2009) ensured that participants were able to correctly identify /s/ and / $/$ / when frequency compression was enabled. If the participant was unable to identify these phonemes correctly, it was assumed that the frequency compression setting was too high and the compression ratio was reduced accordingly.

In a study of 15 children (between the ages of 5 and 13 years old) with moderate hearing loss, Wolfe et al (2010, 2011) also found evidence of significant improvements at a group level in the perception of some high frequency speech sounds with the use of FC. As individual data were not presented, it is difficult to ascertain how much the degree of benefit from FC varied between listeners. 
Ellis \& Munro, Frequency compression hearing aids

The purpose of the present study is to further investigate whether, following a period of auditory

acclimatisation, FC leads to improvements in speech perception and self-report outcome measures in experienced adult hearing aid users.

\section{Auditory acclimatisation}

Auditory acclimatisation (or perceptual learning) was defined by Arlinger et al (1996, ps87) as ' $a$ systematic change in auditory performance with time, linked to a change in the acoustic information available to the listener. It involves an improvement over time that cannot be attributed purely to task, procedural or training effects.' In order to assess benefit from hearing devices, it is necessary to have an understanding of the time course and magnitude of auditory acclimatisation, along with the degree of individual variation in this process. This process is distinct from the audiological practice of slowing increasing amplification, or the strength of a particular signal processing algorithm, over time.

To date, there has been little research into how different signal processing strategies affect the acclimatisation process. Previous studies investigating the effect of FC on the speech perception scores of adult listeners have always provided an acclimatisation period (of at least 3 weeks) prior to testing. However, baseline tests, immediately after FC was enabled, were not conducted (see for example: Simpson et al, 2005, 2006; Glista et al, 2009). Thus, whilst it has been assumed that experienced adult hearing aid users require time to acclimatize to $\mathrm{FC}$, there is currently no empirical evidence to support this idea.

Whilst acclimatisation to frequency compression hearing aids in adult listeners has not yet been investigated, there has been some research of acclimatisation to frequency compression hearing aids in pediatric hearing aid users. Wolfe et al (2010) provided fifteen children, aged 5-13 years old, with 
Ellis \& Munro, Frequency compression hearing aids

moderate to moderately-severe hearing losses, with bilateral frequency compression hearing aids. A within-subjects design was employed, in which the children wore the hearing aids with and without frequency compression enabled for six weeks in each condition (the order was counterbalanced). After each six week period, aided thresholds and performance on a number of speech tests (including plural recognition, sentence in noise recognition and a speech-sound detection task) were assessed. The results showed that FC led to significant improvements in performance on the plural recognition task and in the detection of two of the six stimuli (/asa/ and /ada/) used in the speech-sound detection task. FC did not lead to statistically significant improvements in the recognition of sentences in noise. The participants were then re-tested after 6 months use of FC (Wolfe et al, 2011). The findings show little evidence of changes over time to the perception of frequency-compressed speech, the only significant improvement being a reduction in the detection threshold for one (/ada/) of the six stimuli used in the speech-sound detection task. Small improvements in the detection of another of the stimuli (/ata/) and in sentence in noise recognition meant that the scores obtained after six months of FC use were significantly better than scores obtained after six weeks use of the hearing aids with FC disabled.

The results of Wolfe et al $(2010,2011)$ potentially provide some evidence of acclimatisation to frequency compressed speech. However, baseline measures were not obtained immediately after fitting so it is impossible to ascertain from the data how much acclimatisation may have taken place within the initial six weeks of use. Previous research of acclimatisation to conventional hearing aids has shown that significant changes may take place in the initial six weeks post fitting (Gatehouse, 1992; Munro and Lutman, 2003). A further issue that may influence the validity of these results stems from the fact that there was no control group for the measurements taken after 6 months of frequency compression use. It is possible, therefore, that the differences in scores may be due to maturational effects (see for example, Mahon and Crutchley, 2006, for details of age related changes in receptive vocabulary) or to acclimatisation to other features of the hearing aids, rather than to frequency compression specifically. In particular, maturational effects may have influenced scores on the BKB-SIN as the stimuli used contain 
Ellis \& Munro, Frequency compression hearing aids

large amounts of contextual information, which a child may be better able to use as they develop and their

receptive vocabulary improves. Furthermore, no individual data are presented, so the level of individual differences in the magnitude of acclimatisation to frequency compression cannot be assessed.

More recently, Glista et al (2012) report 6 case studies of acclimatisation to FC in children aged 11-18 years old. Each participant demonstrated a different pattern of results, however, limited evidence for two different patterns of acclimatisation (gradual linear or sudden non-linear after 6-10 weeks of FC use) was found. The varied nature of the results and the use of a case-study approach mean it is difficult to determine the extent to which these findings can be applied to the general population. Furthermore, the sudden non-linear delayed pattern of acclimatisation was primarily limited to performance in a particular task (/s/ - / $/$ / discrimination), so the extent to which this pattern may generalize to real-world acclimatisation is also unclear.

The amount of individual variation in acclimatisation has important implications for clinical practice, specifically regarding how best to counsel patients and the appropriate timescale in which to schedule follow-up appointments. Thus, in addition to investigating acclimatisation to frequency compression hearing aids, the present study will also attempt to identify possible predictors of benefit from frequency compression.

\section{METHOD}

\section{Participants}

Twelve adults ( 65 to 84 years old, median $=75.5$ years old $)$ with symmetrical bilateral moderate-tosevere high-frequency sensorineural hearing loss (no asymmetry of $>20 \mathrm{~dB}$ between the left and right ears 
Ellis \& Munro, Frequency compression hearing aids

at the same frequency), and $>1$ year hearing aid experience took part in the study. In order to detect a mean difference over time of $5 \%$ ( $1 \mathrm{sd} \pm 5$, a difference regarded as clinically significant by the authors), a minimum of 10 participants were required for a statistical power $>80 \%$ on a 2 -tailed paired statistical test at a significance level of 5\%. Ethical approval was granted by Greater Manchester West Research Ethics Committee on behalf of the NHS (Approval code: 10/H1014/44). Participants were compensated for the travel but were not paid for taking part in the study. Participants received free hearing aids, however, they were informed that the same devices could have been obtained (free of charge) from the National Health Service, so this is likely not to have served as an incentive for participation.

\section{Table 1 here}

One participant (002) was not a native English speaker, however had been resident in the UK for 15 years. As the analyses focused on additional benefit from frequency compression, as opposed to mean scores, this participant was accepted onto the study on the basis that their level of English language proficiency was unlikely to change over the course of the trial. Details of each participants hearing loss and relevant history are provided in Table 1 . Hearing thresholds were measured using pure tone audiometry and the presence of dead regions was assessed using the TEN test (Moore et al, 2000; 2004). Frequencies between 250 and $8000 \mathrm{~Hz}$ were assessed using a TEN noise level of at least $+10 \mathrm{~dB}$ SL.

\section{Hearing Aid Fitting and Verification}

Participants were fitted bilaterally with Phonak Naida V SP hearing aids programmed to match NAL-NL2 prescription targets (Keidser et al, 2011) with frequency compression disabled. Table 2 shows prescription targets and best match (after fine tuning).

Table 2 here 
Ellis \& Munro, Frequency compression hearing aids

Once the initial gain settings were finalized (after fine tuning to meet targets), frequency compression was enabled. The Phonak fitting software (iPFG, 2.6) allows the audiologist to alter the cut-off point and compression ratio together. In almost all cases, the frequency compression setting enabled was stronger than the setting recommended in the fitting software (i.e., a lower cut off frequency and higher compression ratio). In order to ensure than the frequency compression setting was appropriate, a number of criteria were assessed. The frequency compression setting that allowed the audibility of the widest possible bandwidth whilst maintaining a cut-off frequency of $>2 \mathrm{kHz}$ (in order not to adversely affect sound quality) was selected. Participants were then asked to discriminate between recordings of /s/ and /J/ spoken by a recorded female talker using a same-different procedure. If the participant was unable to consistently discriminate between these stimuli (i.e. to be correct $100 \%$ of the time after 5 presentations of the two stimuli), or reported problems with the sound quality (for example, a 'lisping' quality), a weaker frequency compression setting (reflected in a higher cut off frequency and lower compression ratio) was applied. The selected frequency compression settings are shown in Table 1, along with details of the setting recommended by the manufacturer. The frequency compression setting was then disabled and participants wore the hearing aids at home for one week in order to determine if any alterations to the hearing aid settings were required. Three participants requested reductions in the low frequency gain of between 2 and $6 \mathrm{~dB}$. No further adjustments were made during the trial.

Automatic program selection and noise reduction features were disabled for the duration of the study, as were the manual volume controls. Hearing aid usage was monitored throughout the trial using the data logging feature of the hearing aids. All participants wore the devices for an average of at least 8 hours per day throughout the trial.

\section{Study Design}


Ellis \& Munro, Frequency compression hearing aids

After fitting the hearing aids, an A-B-A design was used where $\mathrm{A}=$ frequency compression disabled, and $\mathrm{B}=$ frequency compression enabled. All participants wore the hearing aids with frequency compression disabled first (for a mean duration of 3.6 weeks, $\mathrm{SD}=0.81$ ), then enabled (for a mean duration of 6.6 weeks, $\mathrm{SD}=0.95$ ) then disabled again (for a mean duration of 4.0 weeks, $\mathrm{SD}=2.75$ ). Thus, the participants wore the devices at home for approximately six to seven weeks with each setting. The variation in time spent in each condition was caused by the practicalities of fitting in appointments around other activities in the participants' lives. Participants were not told whether frequency compression was enabled or disabled at any point in the trial. Double blinding was not possible as one researcher was responsible for programming the hearing aids and collecting all subsequent test data.

Participants completed a number of speech tests and questionnaires (see details later) at the end of each trial period. In order to compare the benefit obtained with and without frequency compression, the mean of the two sets of scores obtained without frequency compression were compared to performance with frequency compression enabled. Participants also completed speech tests and questionnaires at three additional sessions that took place at 0,1 and 3 weeks after frequency compression was enabled in order to investigate the effect of short-term acclimatisation.

All testing was conducted in a quiet room, acoustically representative of a normal living room (with reverberation times of $<0.3 \mathrm{~s}$ measured in octave intervals from 0.125 to $8 \mathrm{kHz}$ ). Prior to fitting the study hearing aids, the participants attended two familiarization sessions in which they were introduced to the speech tests to be used throughout the study. In these sessions, participants followed the same testing procedures and similar (but not identical) stimuli to those that would eventually be used in the experimental test sessions. This was in order to control for the effects of procedural learning on task performance. In addition to attending testing sessions, participants were asked to spend one hour a week listening to an audio book whilst at the same time following the text. This was done as an aide to help them acclimatize to their new hearing devices. An investigation of the effect of listening to an audiobook 
Ellis \& Munro, Frequency compression hearing aids

on speech perception outcomes was not an aim of the study. We were not interested in training the

participants, merely in making sure that they engaged in 'active listening', at least some of the time, with their hearing aids. We do not envisage that this had a significant effect on outcomes (nor do we consider it to have constituted 'training'), but ensured that listeners spent similar amounts of time on the task to control for this possibility. Participants were provided with audio equipment where required and asked to keep a diary detailing the time they spent listening to the audiobook. All participants completed this task with little variation in the amount of time spent doing it.

\section{Outcome measures}

\section{VCV recognition}

Nonsense syllable recognition tests, in both quiet and noise, were administered so as to reduce the influence of contextual cues on performance. Stimuli consisted of 20 nonsense syllables in /i/ - consonant - /i/ context (for example, /iki/) spoken by a female talker. The stimuli were taken from the IHR VCV test package (Faulkner, 1998; IHR Products, Nottingham) and included the consonants: /b/, /d/, /g/, /w/, /j/, /r/, /l/, /v/, /z/, /dz/, /m/, /n/, /p/, /t/, /k/, /f/, / / /, /s/, / / / and /ts/. Consonants may be more difficult to identify in a VCV context compared to a CVC context, however in order to better facilitate comparisons to the results of previous studies (for example, Simpson et al, 2006), VCV words were presented in the present study.

Stimuli were presented via a loudspeaker situated one meter from the listener at zero degrees azimuth. The level at which the stimuli were presented was set individually for each listener (between 55 and 70 $\mathrm{dB}$ SPL) to minimize the risk of floor and/or ceiling effects and ensure that there was scope for improvement over the trial period. The level was set at the point at which participants achieved mean scores of between 65 and $88 \%$ correct without frequency compression enabled. One participant achieved 
Ellis \& Munro, Frequency compression hearing aids

scores above this level (91\%) and three participants were only able to achieve scores below this range even at a presentation level of $70 \mathrm{~dB}$ SPL $(48 \%, 54 \%$ and $63 \%$ respectively). The presentation level was fixed between test sessions.

Each test run consisted of one practice block (not scored) and four test blocks of 20 presentations each (one presentation of each VCV token). Each token was presented once per block in random order. Participants were given a response sheet with a matrix showing each of the 20 possible consonants. Participants pointed to the sound corresponding to the speech stimulus they heard and responses were then recorded on the computer by the experimenter. It is possible that this process may have introduced some bias, however, most of the participants were unfamiliar with computers and preferred the experimenter to input the responses.

The procedure for testing VCV recognition in noise (multitalker babble) was almost identical to that undertaken in quiet. Again, stimuli were presented via a loudspeaker situated one meter from the listener at zero degrees azimuth. The level of presentation varied between participants but was the same as the level used in quiet. The level of the VCV stimulus was maintained and noise was added to create the desired speech to noise ratio (SNR). The SNR was determined on an individual basis to prevent ceiling or floor effects (and varied from +2 to -2 SNR, with the exception of one participant, 008, who was tested with an SNR of +10 due to floor effects). An SNR that resulted in a 10-20\% decrease in score when compared to performance on the VCV in quiet test was selected.

\section{Sentence in Noise recognition}

A sentence in noise test was administered to give an indication of speech perception performance in a context similar to that experienced in real life. Lists of 10 Institute of Electrical and Electronics Engineers (IEEE) sentences (Rothauser et al, 1969; for example, 'His shirt was clean but one button was gone') 
Ellis \& Munro, Frequency compression hearing aids

spoken by a female talker were presented in a background of 20 talker babble noise (Auditec, St Louis,

USA). The stimuli were again presented via a loudspeaker situated at head height one meter from the listener at zero degrees azimuth. The presentation level of the sentences remained constant throughout each list but the level of the noise varied from +15 to $-12 \mathrm{~dB}$ in a fixed order, one sentence at each SNR, similar to the method recommended by Wilson et al (2007). Sentences were presented at $55 \mathrm{~dB}$ SPL for all participants except one (participant 008) who was unable to complete the task at this level. For this listener, sentences were presented at $65 \mathrm{~dB}$ SPL. The participants' task was to repeat each sentence back to the experimenter and the percentage of keywords ( 5 per sentence) correctly recalled was calculated. Three sentence lists were administered on each occasion of testing. The same sentences lists were used for all participants but were randomly allocated to different test sessions.

\section{Self-report questionnaires}

The Glasgow Hearing Aid Benefit Profile (GHABP; Gatehouse, 1999) is a self-report questionnaire assessing six dimensions consisting of initial disability, initial handicap, hearing aid use, hearing aid benefit, residual disability and satisfaction. Each dimension was assessed in relation to four pre specified listening situations (if they occur in the participant's life and cause them difficulty) with the option of including up to four additional listening situations specified by the respondent. The GHABP has been used with success to examine patterns of benefit in linear and nonlinear hearing aid fittings (Gatehouse et al, 2006a), the effect of counseling on perceived hearing aid benefit (Kemker and Holmes, 2004) and on acclimatisation to the fitting of monaural hearing aids (Munro and Lutman, 2004). The GHABP was administered by the experimenter as a structured interview. Ratings for the final three dimensions were obtained at four intervals, giving two measures with frequency compression disabled (after three and six weeks of use with this setting) and two measures with frequency compression enabled (at one and six weeks post fitting). Ratings for the two initial dimensions, based on participants' experiences with their 
Ellis \& Munro, Frequency compression hearing aids

own hearing aids, were obtained at the first instance of administering the questionnaire, that is at three weeks post fitting of the study hearing aids.

The Speech, Spatial and Qualities (SSQ) Hearing Scale (Gatehouse and Noble, 2004) is a self-report measure consisting of three subscales. (speech perception, spatial hearing and quality of sound). Each subscale is comprised of a series of listening situations which the respondent marks out of 10 , where a score of 10 indicates complete ability and a score of zero indicates minimal ability (Noble and Gatehouse, 2006). A total of 50 listening situations are rated. The SSQ Hearing Scale was administered in the form of a structured interview. The SSQ Hearing Scale was administered on the same four occasions as the GHABP.

\section{Analysis}

In order to investigate the effect of acclimatisation to, and performance with, FC, a series of analyses were carried out. At a group level, the effect of session number on performance (FC0 x FC6, where FC0 refers to tests administered immediately after FC was enabled and FC6 refers to testing conducted after 6 weeks of FC use) and of benefit from frequency compression (FC6 x the mean score from the two No FC testing sessions) were examined using a series of paired samples t-tests. The difference in performance between scores at FC6 and scores at the final No FC test session was also investigated. Prior to conducting these analyses, data were checked to ensure that the assumptions of normality were met. This was accomplished via visual inspection of histograms. In addition, confusion matrices were generated using the results obtained in the nonsense syllable tests. Where a change in consonant recognition or confusion greater than $10 \%$ (deemed by the authors to be a clinically-significant difference) was observed, these results are reported below. Changes of a lesser magnitude are not reported. 
Ellis \& Munro, Frequency compression hearing aids

\section{RESULTS}

\section{Acclimatisation}

\section{VCV Recognition in Quiet}

An inspection of the group means (Figure 1, top panel) shows that performance on the VCV recognition in quiet test at FC6 was slightly higher than that at FC0 with a mean percentage correct of $80.5 \%$ ( $\mathrm{SD}=$ 14.60) at FC6 compared to $78.5 \%(\mathrm{SD}=14.60)$ at FC0. The results of a paired samples t-test confirmed that there was no significant effect of session number on performance $(\mathrm{t}[23]=-1.44, \mathrm{p}=0.163)$.

\section{Figure 1 here}

The individual data (presented in Figure 1) show that 7 participants performed better at FC6 than at FC0, 5 participants scored within $1.5 \%$ (this value having been arbitrarily selected) of their FC0 score at FC6 and no participants performed more poorly at FC6 than at FC0. An analysis of consonant confusions revealed large improvements (over 10\%) in the correct identification of /f/ and / $\theta /$ and an accompanying decrease in the proportion of times that these phonemes were confused for $/ \mathrm{s} /$. A decrease in the proportion of times that $/ \mathrm{t} 3 /$ was confused for $/ \mathrm{t} /$ was also evident.

\section{VCV Recognition in Noise}

An inspection of the group means (shown in Figure 1, middle panel) shows that performance on the VCV recognition in noise test at FC6 was better than that at FC0 with a mean percentage correct of 70.2\% (SD $=15.71)$ at FC6 compared to $68.7 \%(\mathrm{SD}=14.71)$ at FC0. The results of a paired samples t-test indicated that there was a significant effect of session number on performance $(\mathrm{t}[23]=-2.14, \mathrm{p}=0.044)$. 
Ellis \& Munro, Frequency compression hearing aids

The individual data (presented in Figure 1) show that 5 participants performed better at FC6 than at FC0, 7 participants scored within $1.5 \%$ of their FC0 score at FC6 and no participants performed more poorly at FC6 than at FC0. Large improvements (over 10\%) in the correct identification of $/ \mathrm{n} /, / \mathrm{l} /$ and $/ \theta /$ and an accompanying decrease in the proportion of times that $/ \theta /$ and /f/ were confused for $/ \mathrm{s} /$ were observed at

FC6 compared to FC0. In addition, a decrease in the proportion of times that $/ \mathrm{t} 3 /$ was confused for $/ \mathrm{t} / \mathrm{is}$ also evident. The proportion of times that $/ \mathrm{l} /$ was mistaken for $/ \mathrm{m} /$ also decreased over time. However, an increase in confusions between $/ \mathrm{m} /$ and $/ \mathrm{n} /$ over time is apparent.

\section{Sentence in Noise Recognition}

An inspection of the group mean percentage correct scores (presented in Figure 1, lower panel) show that scores obtained on the sentence in noise recognition test at FC6 were very similar to those obtained at FC0 with mean scores of 56.5\% (SD = 12.67) and 55.2\% ( $\mathrm{SD}=14.43)$, respectively. The results of paired samples t-test indicate that there was no significant effect of session number on performance $(\mathrm{t}[35]=$ $0.82, \mathrm{p}=0.415)$

An inspection of the individual results (presented in Figure 1) show that 5 participants obtained better scores at FC6 than at FC0, 2 participants scored within $1.5 \%$ of their FC0 scores at FC6 and 5 participants performed worse at FC6 than at FC0.

\section{Benefit from FC}

\section{VCV Recognition in Quiet}

An inspection of the group means (displayed in Figure 2, upper panel) shows that mean percentage correct on the VCV recognition in quiet test with FC enabled $(80.5 \%, \mathrm{SD}=14.60)$ was higher than No FC $(73.6 \%, S D=14.21)$. The results of a paired samples t-test confirm that there was a significant effect 
Ellis \& Munro, Frequency compression hearing aids

of signal processing strategy on performance $(\mathrm{t}[23]=-2.87, \mathrm{p}=0.001)$. The benefit obtained from FC remains significant when compared to performance in the final No FC test session only $(\mathrm{t}[23]=-2.19, \mathrm{p}=$ 0.039).

Figure 2 here

The individual data (presented in Figure 2) show that 7 participants performed better with FC enabled than without FC, 3 participants obtained similar results (with a difference of less than $1.5 \%$ ) with both signal processing strategies and 2 participants performed more poorly with FC than with No FC. Large improvements (over 10\%) in the correct identification of /s/ and /z/ and an accompanying decrease in the proportion of times that $/ \mathrm{s} /$ was mistaken for $/ \mathrm{f} /$ and $/ \theta /$ and that $/ \mathrm{z} /$ was mistaken for $/ \mathrm{v} /$ when FC was enabled were observed, compared to when it was disabled. The percentage of times that $/ \theta /$ was confused with /f/ also decreased when frequency compression was enabled.

\section{VCV Recognition in Noise}

An inspection of the group means (shown in Figure 2, middle panel) shows that performance on the VCV recognition in noise test in the FC condition was better than that in the No FC condition with mean percentage correct scores of $70.2 \%(\mathrm{SD}=15.71)$ and $61 \%(\mathrm{SD}=13.63)$, respectively. The results of a paired samples t-test indicate that the effect of signal processing strategy on performance was significant $(\mathrm{t}[23]=-5.37, \mathrm{p}<0.001)$. The benefit obtained from $\mathrm{FC}$ remains significant when compared to performance in the final No FC test session only $(\mathrm{t}[23]=-2.21, \mathrm{p}=0.038)$.

The individual data (presented in Figure 2) show that 9 participants performed better with FC enabled than without it and 2 participants performed more poorly with FC when compared to their performance in the No FC condition. Large improvements (over 10\%) were observed in the correct identification of /d/, /z/, /k/, /s/ and /v/ and a large decrease in confusions between /r/ and /w/, /z/ and /v/, /k/ and /p/, /s/ and /f/ 
Ellis \& Munro, Frequency compression hearing aids

and $/ \mathrm{s} /$ and $/ \theta /$ when frequency compression was enabled. However, the use of frequency compression also resulted in an increase in confusions between $/ \mathrm{m} /$ and $/ \mathrm{n} /$.

\section{Sentence in Noise Recognition}

The group mean results, presented in Figure 2 (lower panel), indicate that performance on the sentence in noise recognition test with FC enabled was better than that with FC disabled with mean percentage correct scores of 56.5\% $(\mathrm{SD}=12.67)$ and $49.7 \%(\mathrm{SD}=16.89)$, respectively. The results of a paired samples t-test confirm the significance of these results $(\mathrm{t}[35]=-4.42, \mathrm{p}<0.001)$. The benefit obtained from FC remains significant when compared to performance in the final No FC test session only $(\mathrm{t}[23]=-$ $2.75, \mathrm{p}=0.009)$

At an individual level, 10 participants performed better in the FC condition than in the No FC condition, 1 participant obtained similar scores (less than $1.5 \%$ difference) in both conditions and 1 participant performed more poorly when FC was enabled than when it was disabled.

\section{Glasgow Hearing Aid Benefit Profile}

Table 3 shows the group and individual mean scores obtained on 4 of the 5 subscales of the GHABP (the hearing aid usage subscale was not included as participants were asked to wear their hearing aids as much as possible during the trial). The subscales relating to initial disability and initial handicap relate to the participants' experience without the use of a hearing aid and will, therefore, not be discussed further. Scores on the final 3 subscales, relating to residual disability, hearing aid benefit and hearing aid satisfaction were obtained with and without FC. The group mean scores were slightly higher in the FC condition than in the No FC condition on the Hearing Aid Benefit and Satisfaction subscales. Scores on the Residual Disability subscale were very similar in the FC and No FC conditions. A series of paired samples t-tests indicated that none of these group differences reached statistical significance (Hearing Aid 
Ellis \& Munro, Frequency compression hearing aids

Benefit: $\mathrm{t}[11]=0.20, \mathrm{p}=0.849$; Satisfaction: $\mathrm{t}[11]=0.76, \mathrm{p}=0.465$; Residual Disability: $\mathrm{t}[11]=-0.29, \mathrm{p}$ $=0.775)$.

\section{Table 3 here}

\section{Speech, Spatial and Qualities of Hearing Scale}

Table 4 shows the group and individual mean scores obtained in the SSQ. The overall means are presented along with mean scores for each of the 3 subscales of the questionnaire, obtained in both the FC and No FC conditions.

\section{Table 4 here}

An inspection of the mean overall scores (presented in Table 4) indicates that, at a group level, there was very little difference between scores given in the No FC and FC conditions. The results of a paired samples t-test confirm that this difference in score is not significant $(\mathrm{t}[11]=-0.29, \mathrm{p}=0.778)$. Scores in each of the three subscales also vary little between the two conditions with no evidence of statistically significant differences between the conditions in any of the subscales (Speech: $t[11]=0.02, p=0.985$; Spatial: $\mathrm{t}[11]=-1.40, \mathrm{p}=0.188$; Qualities: $\mathrm{t}[11]=1.65, \mathrm{p}=0.126$ ).

\section{DISCUSSION}

The findings of the study show that at a group level, participants obtained additional benefit from frequency compression processing on all of the speech measures. However, scores on the SSQ Hearing Scale and GHABP did not reflect this improvement, with similar ratings being given in both the FC and 
Ellis \& Munro, Frequency compression hearing aids

No FC conditions. In agreement with the findings of previous studies of frequency compression (Simpson et al, 2005; Glista et al, 2009), the degree of benefit obtained from FC varied between individuals. In addition, the findings provide limited evidence of short-term acclimatisation to frequency compressed speech. With the exception of the VCVN test, there was no statistically significant effect of time on overall mean performance on the speech measures although large changes can be seen in the pattern of consonant confusions made.

\section{Acclimatisation to frequency compression}

The results of the consonant recognition in quiet test show a large increase over time in the correct identification of $/ \mathrm{f} /$ and $/ \theta /$, and a corresponding decrease in the percentage of times these phonemes were mistaken for $/ \mathrm{s} /$. These stimuli all contain the majority of their spectral energy in the high frequencies thus it is unsurprising that change over time is evident. It is likely that listeners have been used to hearing degraded versions of these consonants, therefore, the results suggest that they needed time to relearn how to correctly label these novel sounds. This pattern was also observed in results of the consonant recognition in noise test, along with further improvements in the correct identification of $/ \mathrm{n} / \mathrm{and} / \mathrm{l} /$. These improvements were accompanied by an increase in confusions between $/ \mathrm{m} /$ and $/ \mathrm{n} /$ with a corresponding decrease in the number of times that $/ \mathrm{m} /$ was mistaken for $/ \mathrm{l} /$. This change in the pattern of confusions between lower frequency consonants is likely to be as a result of frequency compression affecting high frequency formant transition necessary for the discrimination of these stimuli. It is important to note that whilst the pattern of confusions may have altered over time, there is no evidence of a significant decrease in the correct identification of any phoneme, either in the VCVQ or VCVN tests.

There is no evidence of an increase over time in confusions between /s/ and / $/$ in either task. Previous research has indicated that frequency compression may result in an increase in confusions between these two phonemes (Simpson et al, 2006) and thus the lack of acclimatisation effect seen here may be viewed 
Ellis \& Munro, Frequency compression hearing aids

as surprising. However, the results of the present study show that the mislabeling of /s/ as /J/ occurred only $10.4 \%$ (in the VCVQ test) and $8.3 \%$ (in the VCVN test) of the time immediately after frequency compression was enabled. Therefore, whilst changes in the rate of these confusions did occur (down to $6.3 \%$ and $4.2 \%$ in the VCVQ and VCVN after 6 weeks experience of FC), these changes were relatively small.

This difference between the results of Simpson et al (2006) and the present study is likely to be related to differences in both the audiograms of the listeners and the fitting strategy employed. In the current study, one of the criteria used to assess the suitability of the frequency compression settings was to ensure that the listener was able to discriminate between /s/ and /// (similar to the criteria suggested by Glista et al, 2009). Therefore, the relative lack of confusion between these phonemes, observed in the results of the speech tests, is perhaps unsurprising. However, it appears that this method of determining the frequency compression settings did not eliminate consonant confusions, rather it altered their nature. Specifically, the results show a large increase over time in the correct identification of /f/ and $/ \theta /$, and a subsequent decrease in the percentage of times these phonemes were mistaken for $/ \mathrm{s} /$. The results of Simpson et al (2006) show that few listeners in their study showed evidence of making these confusions. There are two possible explanations for this. The first is that the measurements taken in Simpson et al's (2006) study were obtained 4-6 weeks after fitting of the FC device. Therefore, participants may have made these confusions initially but learned to correct these errors over time. The second reason may be related to the aforementioned differences in the strategy used to select the frequency compression setting. Recent work conducted by Hamilton et al (2011) on listeners with normal hearing suggests that strong frequency compression settings lead to confusions between /s/ and /J/ whilst weaker settings lead to confusions between $/ \theta /$ and $/ \mathrm{s} /$. Again, this would be consistent with the current findings. Further research investigating how best to use phonemic confusion data in the selection of frequency compression settings is required. 
Ellis \& Munro, Frequency compression hearing aids

It could be argued that the changes over time observed in the consonant confusion data are evidence of procedural learning rather than acclimatisation to frequency compression. If this was the case, one would expect to see larger changes over time on performance in the consonant recognition tasks when compared to the sentence in noise task. This is due to the fact that stimuli in the consonant recognition tasks were identical at all sessions, whilst the sentence material varied. However, there was no evidence of changes over time in overall mean performance on any of the tests administered. Furthermore, the changes in the pattern of confusions made in the consonant recognition task are primarily restricted to high frequency consonants, where the effect of frequency compression would be most evident. Had there been evidence of acclimatisation in mean performance in any of the speech tests, scores with frequency compression would have been compared to scores without frequency compression, obtained at the end of the trial. This would have allowed for the separation of procedural learning and acclimatisation to frequency compression.

It is important to note that the current findings apply only to experienced hearing aid users with moderateto-severe high frequency hearing loss. Therefore, whilst evidence of acclimatisation to frequency compression in this population appears to be limited to high frequency consonants only, this is likely to be related to the fact only the high frequency information has been altered relative to the conventional hearing aids to which they are accustomed. Thus, the results of the study pertain only to additional acclimatisation to frequency compression. It is expected that acclimatisation would have a much greater effect on perception in new hearing aid users who need to adapt to all aspects of the signal processing, rather than just frequency compression as was the case in the current study.

Other issues that may affect the generalisability of the results include the fact that it is possible that high levels of variability in performance in the speech tests masked changes over time that would be clinically, if not statistically, significant. Further research, using larger numbers of stimuli and/or participants, is needed to investigate this issue. It may also be the case that factors other than speech recognition are 
Ellis \& Munro, Frequency compression hearing aids

influenced by acclimatisation. It may be interesting for future research to focus on investigating changes over time to listening effort. It is also possible that a greater effect of acclimatisation would have been seen if stimuli had been presented at a lower presentation level, thus making the tests more difficult. However, whether an understanding of acclimatisation to very low level stimuli (and thus unlike most listening situations) would be clinically useful is debatable. Furthermore, the findings of the study relate only to acclimatisation taking place in the initial 6 weeks after frequency compression is enabled. Research by Wolfe et al (2011) suggests that additional, albeit limited, changes may take place later than this. Specifically, improvements in the recognition of the phonemes /s/ and /d/ (reflected in lower thresholds needed to identify these stimuli correctly) occurred between 6 weeks and 6 months of frequency compression hearing aid use. These results were obtained in a pediatric population, thus further research is needed to track long term changes in adult users of frequency compression devices.

\section{Benefit from frequency compression}

Consonant recognition scores, in both quiet and in noise, improved significantly with the use of frequency compression. An examination of the confusion matrices shows that the majority of these improvements were restricted to changes in the pattern of recognition of high frequency stimuli. Specifically, large improvements were seen in the perception of stimuli that contain the majority of their energy in high frequency regions (such as /s/ and /z/) and decreases in confusions between stimuli that are discriminated between on the basis of high frequency formant transitions (such as $/ \mathrm{d} /-/ \mathrm{g} /$ and $/ \mathrm{r} /-/ \mathrm{w} /$ ). This is consistent with the results obtained in previous studies of frequency compression (Simpson et al, 2005; Glista et al, 2009, Wolfe et al, 2010) and is to be expected given that the changes introduced by frequency compression are specific to the high frequencies. The results also indicate that changes in the pattern of consonant recognition are more wide ranging when the stimuli are presented in a background of noise than when they are presented in quiet. The largest changes, in both the VCVN and VCVQ results, were 
Ellis \& Munro, Frequency compression hearing aids

seen with regards to confusions between /v/ and /z/. The phoneme /z/ contains more energy than /v/ in the frequency range above $3 \mathrm{kHz}$, their lower frequency spectral composition being very similar. Thus, the improvements in correct identification of /z/ and the accompanying decrease in confusions with /v/ may indicate that FC provided listeners with high frequency information that they did not have access to with conventional amplification. There has been no previous research on the effect of frequency compression on the perception of consonants in noise, and thus no comparisons can be drawn. The observation that frequency compression seems to provide greater benefit in more difficult listening conditions is likely to be related to the fact that listeners performed more poorly in this task than on the VCV in quiet test, leaving more room for improvement with frequency compression. It also demonstrates that this method of frequency lowering does not lead to adverse effects in noisy situations. Furthermore, there is no evidence of an increase in confusions between /s/ and / $/$ / as observed by Simpson et al (2006). This is likely to be related to difference in how the frequency compression settings were selected, with the ability to discriminate between /s/ and /// being one of the criteria used to judge the suitability of a frequency compression setting in the present study.

The results of the sentence recognition in noise test again showed a significant benefit of frequency compression at a group level. This finding is at odds with the results reported by Wolfe et al (2010) who also measured sentence in noise recognition after 6 weeks experience of frequency compression use but found no evidence of significant improvements compared to scores obtained with conventional amplification. This difference in findings is likely to be related to the nature of the sentence lists used. Wolfe et al (2010) used BKB sentence lists which contain more contextual information than the IEEE sentences used in the present study. It seems reasonable to assume that the additional audible information provided by frequency compression would provide greater benefit in situations where only limited contextual information is available. In such situations, listeners would need to use cognitive resources to compensate for a distorted signal. Future research is needed to investigate the interaction between 
Ellis \& Munro, Frequency compression hearing aids

contextual information and benefit from frequency compression. It is also possible that the difference in

findings relates to variations in participant characteristics between the studies. The participants in Wolfe et al's (2010) study were children who had less severe high frequency losses that the adult listeners in the present study.

Even though frequency compression was shown to provide significant benefit to speech perception, the results of the self-report measures indicated that there was little difference in subjective ratings of the two signal processing methods. Furthermore, at an individual level, ratings given in the questionnaires did not correspond with the degree of benefit obtained from frequency compression in the speech tests. Again, these findings are consistent with those reported by Simpson et al (2006). However, whilst scores on the GHABP and SSQ Hearing Scale did not improve significantly with frequency compression, they did not get significantly worse.

High levels of variability in the data at an individual level meant that parametric statistical analyses could not be used. Therefore, in order to discuss individual differences in benefit obtained from FC, it is suggested that listeners who achieved higher scores in the majority of speech tests (on at least 2 of the 3 tests administered) should be considered good frequency compression users. If this criterion is applied, 10 of the 12 participants showed evidence of obtaining benefit from frequency compression. No participants were significantly disadvantaged by the use of FC on any of the tests administered.

At the end of the trial, all participants chose to keep the study hearing aids and 10 participants preferred to have frequency compression enabled (including 2 of the listeners who obtained no obvious benefit from frequency compression). Of the 2 participants who chose to have the frequency compression disabled after the trial, one had no real preference (in concurrence with his speech perception scores) so decided to 
Ellis \& Munro, Frequency compression hearing aids

stick with conventional processing. The other participant did obtain higher speech perception scores with frequency compression but preferred the quality of sound when frequency compression was disabled.

It could be argued that the fixed A-B-A order effects may have influenced because the two experimental conditions were not counterbalanced. When calculating the benefit provided by frequency compression, the mean scores obtained after 3 and 6 weeks hearing aid use without frequency compression (at the end of each 'A' period) were compared to those obtained after 6 weeks experience of frequency compression use (at the end of the ' $\mathrm{B}$ ' period). If there was a large task learning effect, one may suggest that inclusion of scores obtained at the end of the first 'A' period may have biased the results in favour of frequency compression. To address this issue, the results were recalculated and the scores obtained with frequency compression were compared to the final scores obtained without frequency compression. A significant benefit from frequency compression was still evident for all of the speech tests administered. Again, there was no effect of signal processing condition on ratings given in either the SSQ or the GHABP.

\section{Conclusions}

The results suggest that frequency compression provides significant benefit (above that obtained from conventional amplification) to speech perception for many listeners with moderate-to-severe sensorineural hearing loss, at least on lab-based outcome measures. However, this improvement may not be reflected in subjective ratings of frequency compression. No evidence of a significant disadvantage to any participant from the use of FC was observed.

The findings show that acclimatisation in the first 6 weeks of frequency compression use is limited to changes in the perception of certain high frequency phonemes, in particular a reduction in mislabeling /f/ and $/ \theta /$ as $/ \mathrm{s} /$. The effect of acclimatisation seems to be greatest in difficult listening situations (nonsense syllable recognition in noise). 
Ellis \& Munro, Frequency compression hearing aids

\section{ACKNOWLEDGEMENTS}

This study was funded by an ESRC CASE PhD studentship in conjunction with Phonak AG. The authors are grateful to the participants for donating their time and to Catherine Siciliano, Kathryn Hopkins, Richard Baker and Michael Akeroyd for helpful comments on an earlier draft of this paper. The authors would also like to thank Kathryn Lewis and staff at the Audiology department at Withington Community Hospital, Manchester for help recruiting participants, Keith Wilbraham for technical assistance, Anna Pepler for clinical assistance and Andrea Simpson for her help in getting the project started.

Declaration of interest: The authors report no conflict of interest. The authors alone are responsible for the content and writing of the paper.

\section{REFERENCES}

Arlinger, S., Gatehouse, S., Bentler, R. A., Byrne, D., Cox, R.M. et al (1996) Report of the Eriksholm workshop on auditory deprivation and acclimatisation. Ear Hear, 17, S87-S98.

Ellis, R.J \& Munro, K. J. (under review) Predictors of outcome from frequency compression hearing aids 
Ellis \& Munro, Frequency compression hearing aids in experienced adult hearing aid users

Gatehouse, S. (1992) The time course and magnitude of perceptual acclimatisation to frequency responses: Evidence from monaural fitting of hearing aids. J Acoust Soc Am, 92, 12581268.

Gatehouse, S. (1999) Glasgow Hearing Aid Benefit Profile: Derivation and validation of a client-centred outcome measure for hearing aid services. J Am Acad Audiol, 10, 80-103.

Gatehouse, S. \& Noble, W. (2004) The Speech, Spatial and Qualities of hearing scale (SSQ). Int J Audiol, 43, 85-99.

Gatehouse, S., Naylor, G. \& Elberling, C. (2006a) Linear and nonlinear hearing aid fittings - 1. Patterns of benefit. Int J Audiol, 45,130-152.

Glista, D., Scollie, S., Bagatto, M., Seewald, R., Parsa, V. et al (2009) Evaluation of nonlinear frequency compression: Clinical outcomes. Int J Audiol, 48, 632-644.

Glista, D., Scollie, S. \& Sulkers, J. (2012) Perceptual acclimatisation post nonlinear frequency compression hearing aid fitting in older children. J Speech Lang Hear Res, 55, 1765-1787.

Hamilton, A., Siciliano, C. \& Munro, K. J. (2011, September) Non-linear frequency compression: An analysis of errors and a comparison of auditory training methods with normal hearing adults. Poster presented at the BSA Annual Conference, Nottingham. UK.

Keidser, G., Dillon, H. R., Flax. M., Ching, T. \& Brewer, S. (2011) The NAL-NL2 prescription procedure. Audiol Res doi:10.4081 /audiores.2011.e24

Kemker, B. E. \& Holmes, A. E. (2004) Analysis of prefitting versus postfitting hearing aid orientation using the Glasgow Hearing Aid Benefit Profile (GHABP). J Am Acad Audiol, 15, 311-323.

Mahon, M. \& Crutchley, A. (2006) Performance of typically-developing school-age children with English as an additional language on the British PictureVocabulary Scales II. Child Lang Teach Ther, 22, 333-351. 
Ellis \& Munro, Frequency compression hearing aids

McDermott H. J. (2011) A Technical Comparison of Digital Frequency-Lowering Algorithms Available in Two Current Hearing Aids. PLoS ONE 6(7): e22358. doi:10.1371/journal.pone.0022358

Moore, B. C. J., Huss, M., Vickers, D. A., Glasberg, B. R. \& Alcántara, J. I. (2000) A test for the diagnosis of dead regions in the cochlea. Br J Audiol, 34, 205-224.

Moore, B. C. J., Glasberg, B. R. \& Stone, M. A. (2004) New version of the TEN test with calibrations in dB HL. Ear Hear, 25, 478-487.

Munro, K. J. \& Lutman, M. E. (2003) The effect of speech presentation level on measurement of auditory acclimatisation to amplified speech. J Acoust Soc Am, 114, 484-495.

Munro, K. J. \& Lutman, M. E. (2004) Self-reported outcome in new hearing aid users over a 24-week post-fitting period. Int J Audiol, 43, 555-562.

Noble, W. \& Gatehouse, S. (2006) Effect of bilateral versus unilateral hearing aid fittings on abilities measured by the Speech, Spatial and Qualities of Hearing Scale (SSQ). Int J Audiol, 45, 172-181.

Rothauser, E. H., Chapman, W. D., Guttman, N., Nordby, K. S., Silbiger, H. et al (1969) IEEE recommended practice for speech quality measurements. IEEE Trans Acoust, 17, 227-246.

Simpson, A., Hersbach, A. A. \& McDermott, H. J. (2005) Improvements in speech perception with an experimental nonlinear frequency compression hearing device. Int J Audiol, 44, 281-292.

Simpson, A., Hersbach, A. A. \& McDermott, H. J. (2006) Frequency-compression outcomes in listeners with steeply sloping audiograms. Int J Audiol, 45, 619-629.

Wilson, R. H., McArdle, R. A. \& Smith, S. L. (2007) An evaluation of the BKB-SIN, HINT, QuickSIN and WIN materials on listeners with normal hearing and listeners with hearing loss. $J$ Speech Lang Hear Res, 50, 844-856.

Wolfe, J., John, A., Schafer, E., Nyffeler, M., Boretzki, M. et al (2010) Evaluation of nonlinear frequency compression for school-age children with moderate to moderately severe hearing loss. 
Ellis \& Munro, Frequency compression hearing aids

J Am Acad Audiol, 21, 618-628.

Wolfe, J., John, A., Schafer, E., Nyffeler, M., Boretzki, M. et al (2011) Long-term effects of non-linear frequency compression for children with moderate hearing loss. Int J Audiol, 50, 396-404. 
Ellis \& Munro, Frequency compression hearing aids

\section{LIST OF TABLES}

Table 1. Characteristics of the participants' hearing loss, previous hearing aid use and the non-linear frequency compression settings used in the trial.

Table 2. NAL-NL2 prescription targets and deviation (target - match after fine tuning) expressed in real ear insertion gain $(\mathrm{dB})$ obtained at the initial fitting and final test session.

Table 3. Group and individual mean scores obtained in each subscale of the GHABP with and without frequency compression. The overall mean scores are also presented.

Table 4. Group and individual mean scores obtained in each subscale of the SSQ Hearing Scale with and without frequency compression. The overall mean scores are also presented. 
Ellis \& Munro, Frequency compression hearing aids

\section{Table 1}

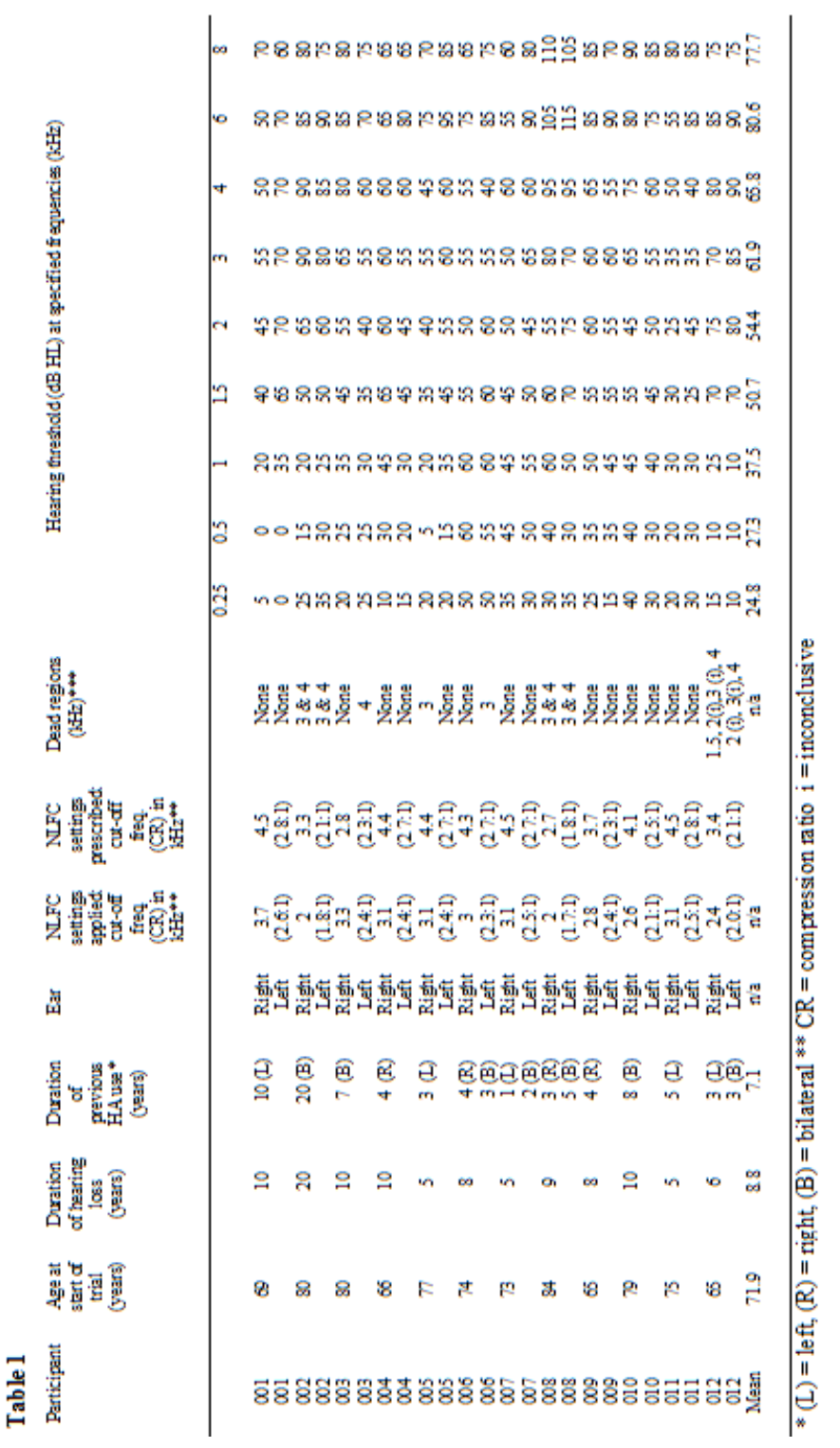


Ellis \& Munro, Frequency compression hearing aids

Table 2

\begin{tabular}{|c|c|c|c|c|c|c|c|c|}
\hline \multirow[t]{2}{*}{$\underset{\text { ( } \mathbb{L} \text { Sevel }}{\text { SPL) }}$} & \multicolumn{8}{|c|}{$\begin{array}{l}\text { Mean targets and deviation (tagget - match in dB) in real ear insertion gain from } \\
\text { target prescribed vaing the NAL-NL2fitting formula. }\end{array}$} \\
\hline & 250 & 500 & 1000 & 2000 & 3000 & 4000 & 6000 & 8000 \\
\hline Target (65) & $\begin{array}{c}3.1 \\
(4.5)\end{array}$ & $\begin{array}{r}29 \\
(4.6)\end{array}$ & $\begin{array}{l}111 \\
(5.6)\end{array}$ & $\begin{array}{l}19.4 \\
(45)\end{array}$ & $\begin{array}{l}227 \\
(5.4)\end{array}$ & $\begin{array}{l}259 \\
(5.2)\end{array}$ & $\begin{array}{l}26.6 \\
(4.7)\end{array}$ & $\begin{array}{l}26.6 \\
(4.5)\end{array}$ \\
\hline $\begin{array}{l}\text { Initial } \\
\text { fitting (65) }\end{array}$ & $\begin{array}{c}24 \\
(4.5)\end{array}$ & $\begin{array}{r}14 \\
(4.9)\end{array}$ & $\begin{array}{l}-27 \\
(4.8)\end{array}$ & $\begin{array}{l}-3.0 \\
(4.7)\end{array}$ & $\begin{array}{r}-20 \\
(7.5)\end{array}$ & $\begin{array}{c}5.1 \\
(104)\end{array}$ & $\begin{array}{c}9.8 \\
(6.6)\end{array}$ & $\underset{(7.8)}{22.5}$ \\
\hline $\begin{array}{l}\text { Initial } \\
\text { fitting(50) }\end{array}$ & $\begin{array}{c}7.7 \\
(7.0)\end{array}$ & $\begin{array}{r}4.6 \\
(4.8)\end{array}$ & $\begin{array}{l}-3.3 \\
(3.8)\end{array}$ & $\begin{array}{l}07 \\
(64)\end{array}$ & $\begin{array}{c}3.8 \\
(8.4)\end{array}$ & $\begin{array}{l}11.2 \\
(10.4)\end{array}$ & $\begin{array}{l}17.4 \\
(6.6)\end{array}$ & $\begin{array}{l}21.6 \\
(6.6)\end{array}$ \\
\hline $\begin{array}{c}\text { Initial } \\
\text { fitting (80) }\end{array}$ & $\begin{array}{l}-0.7 \\
(1.5)\end{array}$ & $\begin{array}{l}-0.6 \\
(20)\end{array}$ & $\begin{array}{l}-3.1 \\
(3.8)\end{array}$ & $\begin{array}{l}-3.9 \\
(60)\end{array}$ & $\begin{array}{l}-28 \\
(69)\end{array}$ & $\begin{array}{c}28 \\
(10.3)\end{array}$ & $\begin{array}{c}53 \\
(69)\end{array}$ & $\begin{array}{l}17.4 \\
(9.5)\end{array}$ \\
\hline $\begin{array}{c}\text { End of trial } \\
(65)\end{array}$ & $\begin{array}{c}0.7 \\
(3.8)\end{array}$ & $\begin{array}{r}24 \\
(5.7)\end{array}$ & $\begin{array}{l}-28 \\
(5.0)\end{array}$ & $\begin{array}{l}-4.5 \\
(42)\end{array}$ & $\begin{array}{l}-10 \\
(5.6)\end{array}$ & $\begin{array}{c}7.7 \\
(87)\end{array}$ & $\begin{array}{l}110 \\
(88)\end{array}$ & $\begin{array}{l}24.2 \\
(7.7)\end{array}$ \\
\hline
\end{tabular}


Ellis \& Munro, Frequency compression hearing aids

Table 3

\begin{tabular}{|c|c|c|c|c|c|c|c|c|}
\hline \multirow[t]{3}{*}{ Participant } & \multicolumn{8}{|c|}{ GHABP Subscale } \\
\hline & \multirow[t]{2}{*}{$\begin{array}{c}\text { Initial } \\
\text { Disability }\end{array}$} & \multirow[t]{2}{*}{ Handicap } & \multicolumn{2}{|c|}{$\begin{array}{l}\text { Hearing Aid } \\
\text { Benefit }\end{array}$} & \multicolumn{2}{|c|}{$\begin{array}{l}\text { Residual } \\
\text { Disability }\end{array}$} & \multicolumn{2}{|c|}{ Satisfaction } \\
\hline & & & FC & No FC & $\mathrm{FC}$ & No $\mathrm{FC}$ & FC & No FC \\
\hline 001 & 31.3 & 25 & 81.3 & 42.7 & 25 & 21.9 & 75 & 63.5 \\
\hline 002 & 25 & 31.3 & 58.3 & 44.8 & 18.8 & 22.9 & 68.8 & 54.2 \\
\hline 003 & 12.5 & 12.5 & 50 & 43.8 & 31.3 & 18.8 & 58.3 & 59.4 \\
\hline 004 & 58.3 & 50 & 43.7 & 53.1 & 37.5 & 40.8 & 62.5 & 58.3 \\
\hline 005 & 31.3 & 31.3 & 81.3 & 75 & 125 & 15.6 & 93.8 & 71.8 \\
\hline 008 & 68.8 & 87.5 & 62.5 & 71.9 & 37.5 & 32.8 & 68.8 & 71.8 \\
\hline 007 & 58.3 & 62.5 & 68.8 & 75 & 18.8 & 18.8 & 75 & 78.1 \\
\hline 008 & 58.3 & 18.8 & 50 & 42.7 & 31.3 & 33.3 & 50 & 55.2 \\
\hline 009 & 87.5 & 58.3 & 68.8 & 62.5 & 25 & 37.5 & 75 & 62.5 \\
\hline 010 & 43.8 & 31.3 & 62.5 & 61.5 & 18.8 & 35.4 & 50 & 53.1 \\
\hline 011 & 58.3 & 68.8 & 56.3 & 90.6 & 40.6 & 15.6 & 58.3 & 93.8 \\
\hline 012 & 43.8 & 56.3 & 50 & 37.5 & 43.8 & 53.1 & 50 & 37.5 \\
\hline Group Mean & 47.4 & 44.3 & 61.1 & 58.4 & 28.2 & 28.9 & 64.9 & 63.1 \\
\hline
\end{tabular}


Ellis \& Munro, Frequency compression hearing aids

Table 4

\begin{tabular}{|c|c|c|c|c|c|c|c|c|}
\hline \multirow[t]{3}{*}{ Participant } & \multicolumn{6}{|c|}{ SSQ Subscale } & \multicolumn{2}{|c|}{ Overall } \\
\hline & \multicolumn{2}{|c|}{ Speech } & \multicolumn{2}{|c|}{ Spatial } & \multicolumn{2}{|c|}{ Qualities } & \multirow[b]{2}{*}{$\mathrm{FC}$} & \multirow[b]{2}{*}{ No FC } \\
\hline & $\mathrm{FC}$ & No FC & $\mathrm{FC}$ & No FC & $\mathrm{FC}$ & No $\mathrm{FC}$ & & \\
\hline 001 & 7.4 & 7.0 & 7.3 & 7.2 & 8.5 & 8.3 & 7.7 & 7.5 \\
\hline 002 & 6.0 & 5.9 & 8.0 & 7.6 & 7.6 & 7.0 & 7.2 & 6.8 \\
\hline 003 & 7.3 & 5.9 & 7.6 & 6.8 & 7.6 & 7.6 & 7.5 & 6.7 \\
\hline 004 & 6.8 & 6.0 & 7.6 & 7.2 & 8.6 & 8.5 & 7.7 & 7.2 \\
\hline 005 & 8.6 & 8.7 & 10 & 9.9 & 9.8 & 9.8 & 9.5 & 9.5 \\
\hline 008 & 7.8 & 7.6 & 8.6 & 7.7 & 9.1 & 9.1 & 8.5 & 8.1 \\
\hline 007 & 7.8 & 6.7 & 7 & 5.6 & 7.9 & 7.3 & 7.6 & 6.5 \\
\hline 008 & 27 & 5.2 & 4.4 & 7.4 & 9.1 & 8.3 & 5.4 & 7.0 \\
\hline 009 & 6.1 & 4.6 & 8.8 & 7.2 & 9.1 & 7.8 & 8.0 & 6.5 \\
\hline 010 & 5.9 & 6.3 & 6.1 & 6.1 & 7.9 & 7.8 & 6.8 & 6.8 \\
\hline 011 & 6.2 & 7.6 & 4.6 & 6.8 & 7.8 & 8.6 & 6.2 & 7.6 \\
\hline 012 & 5.2 & 5.5 & 7.9 & 8.0 & 7.5 & 6.8 & 6.9 & 6.8 \\
\hline Group Mean & 6.5 & 6.5 & 7.3 & 7.8 & 8.4 & 8.1 & 7.4 & 7.5 \\
\hline
\end{tabular}

$\mathrm{FC}=$ frequenc/ compression 
Ellis \& Munro, Frequency compression hearing aids

\section{LIST OF FIGURES}

Figure 1. Group and individual mean scores (percentage correct) at FC0 (white column) and FC6 (shaded column). Error bars show \pm 1 standard error and statistically significant results are marked with an asterisk. Top panel: VCV in quiet test; Middle panel: VCV in noise test, Bottom panel: Sentence in noise recognition test.

Figure 2. Group and individual mean scores (percentage correct) with FC (white column) and without FC (shaded column). Error bars show \pm 1 standard error and statistically significant results are marked with an asterisk. Top panel: VCV in quiet test; Middle panel: VCV in noise test, Bottom panel: Sentence in noise recognition test. 
Ellis \& Munro, Frequency compression hearing aids

Figure 1
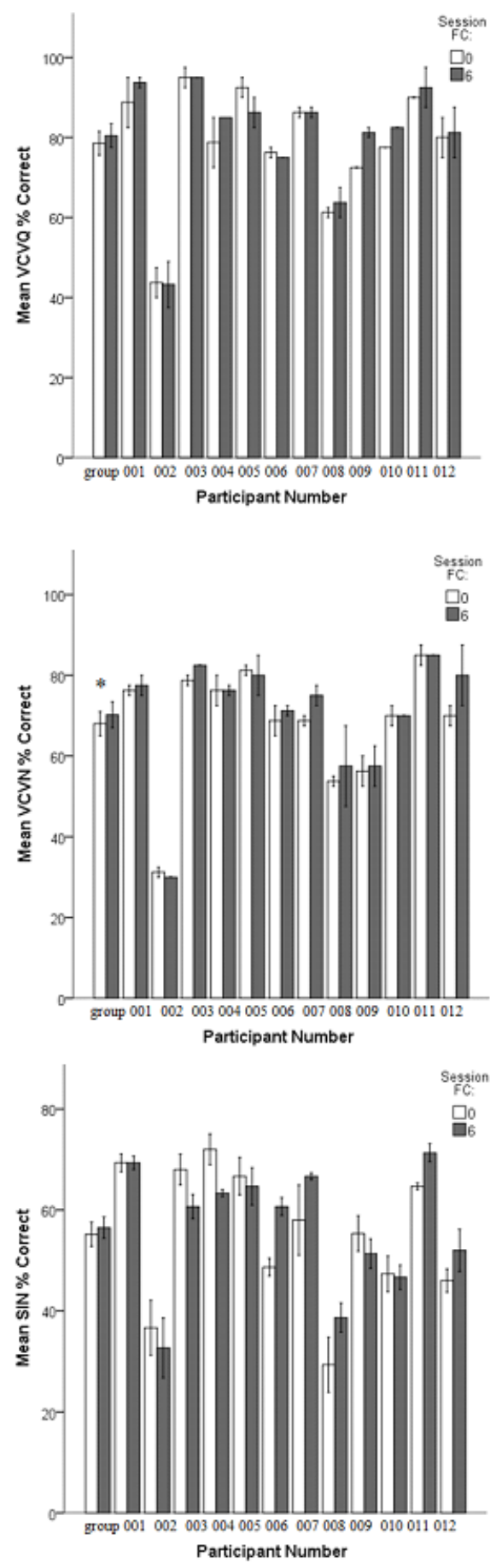
Ellis \& Munro, Frequency compression hearing aids

Figure 2
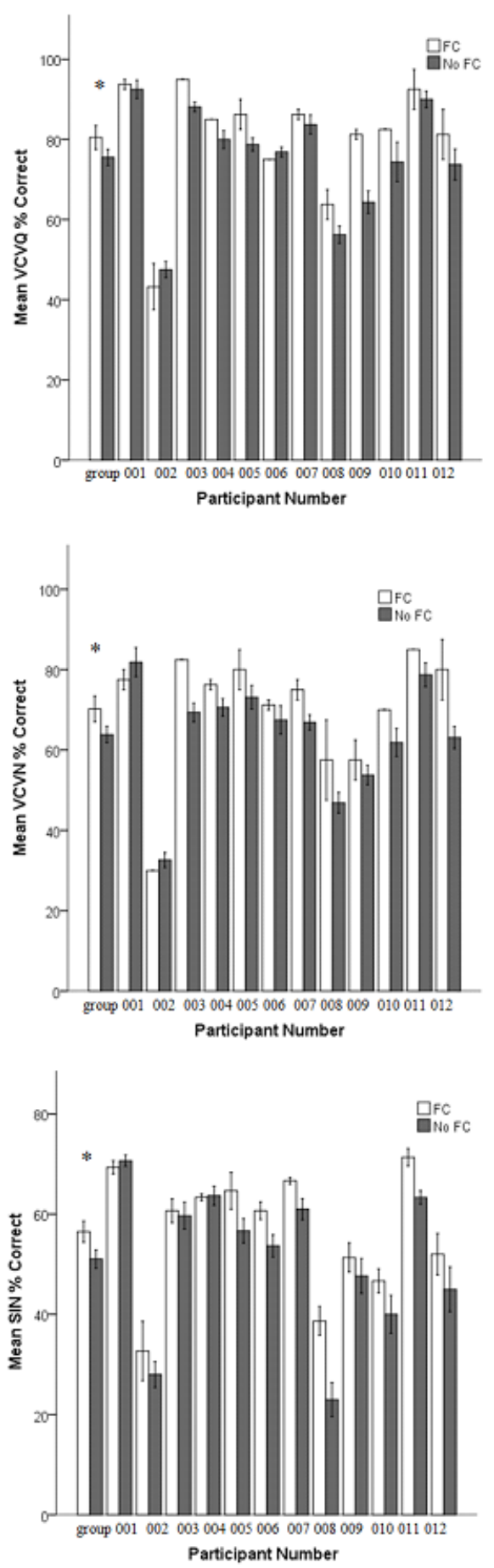